\title{
Modelling mucociliary clearance
}

\author{
D.J. Smith ${ }^{\mathrm{a}, \mathrm{b}, \mathrm{c}, *}$, E.A. Gaffney ${ }^{\mathrm{c}, \mathrm{d}}$, J.R. Blake ${ }^{\mathrm{a}, \mathrm{c}}$ \\ a School of Mathematics, University of Birmingham, Edgbaston, Birmingham B15 2TT, UK \\ ${ }^{\mathrm{b}}$ Reproductive and Child Health, Medical School, University of Birmingham, Edgbaston, Birmingham B15 2TT, UK \\ ' Centre for Human Reproductive Science, Birmingham Women's Health Care NHS Trust, Metchley Park Road, Edgbaston, Birmingham B15 2TG, UK \\ ' Centre for Mathematical Biology, Mathematical Institute, University of Oxford, 24-29 St. Giles', Oxford OX1 3LB, UK
}

\section{A R T I C L E I N F O}

\section{Article history:}

Accepted 12 March 2008

\section{Keywords:}

Morphology

Pseudohypoaldosteronism

Internal mechanics

Cilia

Surface liquid

Mucus

Metachronism

Modelling

\begin{abstract}
A B S T R A C T
Mathematical modelling of the fluid mechanics of mucociliary clearance (MCC) is reviewed and future challenges for researchers are discussed. The morphology of the bronchial and tracheal airway surface liquid (ASL) and ciliated epithelium are briefly introduced. The cilia beat cycle, beat frequency and metachronal coordination are described, along with the rheology of the mucous layer. Theoretical modelling of MCC from the late 1960s onwards is reviewed, and distinctions between 'phenomenological', 'slender body theory' and recent 'fluid-structure interaction' models are explained.

The ASL consists of two layers, an overlying mucous layer and underlying watery periciliary layer (PCL) which bathes the cilia. Previous models have predicted very little transport of fluid in the PCL compared with the mucous layer. Fluorescent tracer transport experiments on human airway cultures conducted by Matsui et al. [Matsui, H., Randell, S.H., Peretti, S.W., Davis, C.W., Boucher, R.C., 1998. Coordinated clearance of periciliary liquid and mucus from airway surfaces. J. Clin. Invest. 102 (6), 1125-1131] apparently showed equal transport in both the PCL and mucous layer. Recent attempts to resolve this discrepancy by the present authors are reviewed, along with associated modelling findings. These findings have suggested new insights into the interaction of cilia with mucus due to pressure gradients associated with the flat PCL/mucus interface. This phenomenon complements previously known mechanisms for ciliary propulsion. Modelling results are related to clinical findings, in particular the increased MCC observed in patients with pseudohypoaldosteronism. Recent important advances by several groups in modelling the fluid-structure interaction by which the cilia movement and fluid transport emerge from specification of internal mechanics, viscous and elastic forces are reviewed. Finally, we discuss the limitations of existing work, and the challenges for the next generation of models, which may provide further insight into this complex and vital system.
\end{abstract}

(ㄷ) 2008 Elsevier B.V. All rights reserved.

\section{Introduction}

\subsection{The airway surface liquid}

The airways of the lung are protected from inhaled dust, bacteria and other harmful substances by an extremely thin surface liquid (ASL), normally from 5 to $10 \mu \mathrm{m}$ deep. The ASL consists of at least two layers: the upper layer consists of highly viscous and non-Newtonian mucus, mainly composed of water (approximately $98 \%$ ), salt (1\%) and glycosylated mucin proteins (1\%), secreted by specialised cells (for review of recent research of mucin secretion, see Davis and Dickey, 2008 and Davis and Lazarowski, in this volume). The lower layer is a watery lubricating fluid, or 'periciliary liquid' (PCL). The origin of the PCL is not known, but is hypothe-

\footnotetext{
* Corresponding author at: School of Mathematics, University of Birmingham, Edgbaston, Birmingham B15 2TT, UK.

E-mail address: D.J.Smith.2@bham.ac.uk(D.J. Smith).
}

sised to originate from osmosis across the epithelium (Sleigh et al., 1988). In order to remove harmful material trapped by the mucus, the layer is transported along the airways and out of the lungs by the activity of a dense mat of microscopic cilia, whereupon it is swallowed or expectorated. Typical mucus velocity values observed in experiment are of the order of tens of microns per second, with the clearance of ASL being modulated over a three-fold range (Knowles and Boucher, 2002). Each mature ciliated cell may have up to 200 cilia, at a density of $6-8 \mu \mathrm{m}^{-2}$ (Sleigh et al., 1988). Fig. 1 shows light microscopy and electron micrograph visualisation of the two-layer ASL structure with beating cilia in human lung tissue culture and in rabbit trachea, respectively. Impairment of MCC is a major contributing factor in cystic fibrosis (CF) lung disease (Boucher, 2007), and in addition to conditions such as asthma and chronic obstructive pulmonary disease (COPD) (Donno et al., 2000; Rogers, 2004, 2005).

Cilia perform an 'effective stroke' in which they beat forwards, probably engage with the mucous layer, and propel it forward. They then perform a 'recovery stroke' in which they return to their initial 

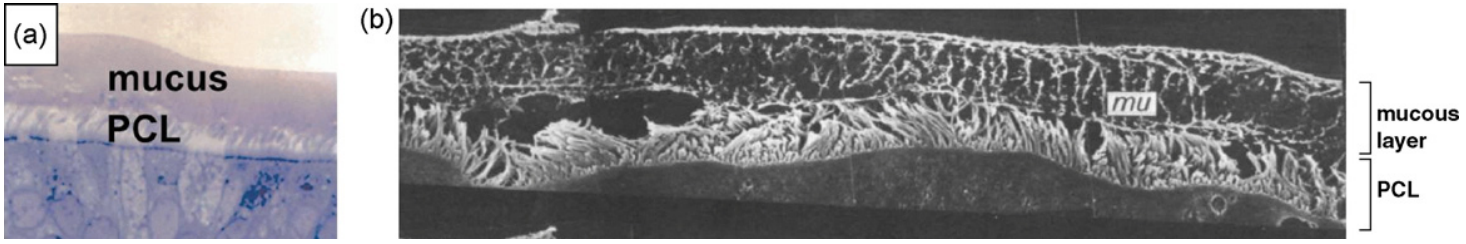

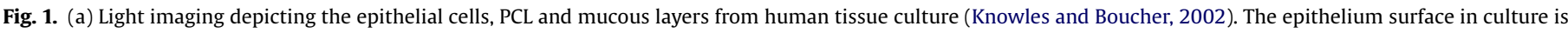

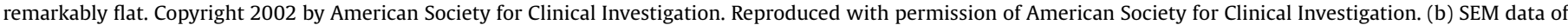

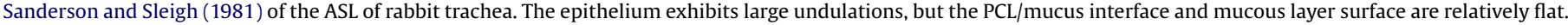
Reproduced with permission of the Company of Biologists.

position but do not drag as much fluid backwards, so that an overall positive flow is produced. A reconstruction of the cilia beat cycle is depicted in Fig. 2a. The classical model holds that the PCL provides a low viscosity environment in which cilia can perform the recovery stroke, while the overlying mucous layer is propelled by the penetration of the cilia tips during the effective stroke. Recently it has been suggested that a further role of the PCL is to prevent the adherence of epithelial mucins with the overlying mucous layer (Knowles and Boucher, 2002). CF epithelia absorb excessive liquid, resulting in a depletion of the PCL, causing a 'double hit': mucous layer tethering to epithelial mucins, and impaired ciliary beating. Experiments on human tissue cultures suggest that secreted liquid preferentially partitions to the mucous layer (Tarran et al., 2001), which may help to preserve efficient cilia-mucus interaction by ensuring that the cilia tips engage with the mucous layer. Excessive mucin secretion by different secretory cell types are likely to be a major cause of the airway plugging associated with fatal asthma (Groneberg et al., 2004).

The bifurcating nature of the lungs results in several orders of magnitude difference in surface area between the distal airways and the trachea (Matsui et al., 1998). Hence as the ASL is propelled from the distal airways to the trachea, it becomes deeper, with volume absorption by the epithelium likely being important in controlling ASL depth.

\subsection{The cilia beat cycle and beat frequency}

Sanderson and Sleigh (1981) used high-speed cinemicroscopy and scanning electron microscopy to visualise the cilia beat frequency and beat cycle, cilium-mucus interaction and metachronal coordination in rabbit tracheal cilia. Example electron micrograph sections are shown in Fig. 1 and their beat cycle reconstruction in Fig. 2. These data were later used in mathematical modelling of MCC, as described in Section 2. Cilia were found to penetrate the mucus to approximately $0.5 \mu \mathrm{m}$ depth. A wide range of cilia beat frequencies $(\mathrm{CBF})$ were reported, apparently modulated by temperature. Marino and Aiello (1982) subsequently measured the CBF of human bronchial cilia as $15.6 \mathrm{~Hz}$ at $34^{\circ} \mathrm{C}$, and found that $\mathrm{CBF}$ fell significantly as temperature decreased.

More recently Chilvers and O'Callaghan (2000) used digital video to visualise the $\mathrm{CBF}$ and cilia beat pattern of human nasal cilia in the absence of mucus. CBF values in the range $11-15 \mathrm{~Hz}$ were reported, and it was found that the beat pattern was planar. This contrasted with observations reported by Sanderson and Sleigh that rabbit tracheal cilia beneath a mucous layer swept in an out-of-plane motion during the recovery stroke. Chilvers et al. (2003) showed that cilia in patients with primary ciliary dyskinesia show a variety of dysfunctional beat patterns. These included: very slow, 'flickering', associated with outer arm and inner/outer arm dynein defects; stiff, low amplitude beating, associated with isolated inner arm or radial spoke defects; and large amplitude circling motion, associated with a microtubule transposition defect. The 'normal beating' reconstruction of Chilvers et al. showed remarkably little difference between the effective and recovery strokes, also contrasting with the study of Sanderson and Sleigh.

For details on the molecular biology of the regulation of CBF, see Salathe (2007) and Davis and Lazarowski (in this volume). Recent modelling, described in Section 2.4, suggests that CBF is a very important parameter in directly regulating mucus velocity and hence clearance, and experiments show that ciliary forces depend linearly on CBF (Teff et al., 2008). In vitro experiments of Maurer et al. (1982) showed that CBF is increased in allergic mucociliary dysfunction. This perhaps suggests that in such conditions the dysfunction is not caused by changes to $\mathrm{CBF}$, but rather that CBF increase represents an adaptive mechanism. Gheber and Priel (1998) examined in detail the effect of viscosity, modulated using dextran polymer, on CBF. They observed a reduction from 11 to $12 \mathrm{~Hz}$ at viscosity $0.001 \mathrm{~Pa}$ s, to around $3 \mathrm{~Hz}$ at $2.0 \mathrm{~Pa}$ s-with cilia exhibiting a remarkable 'operating viscosity range' covering three orders of magnitude. Further detailed study of regulators of $\mathrm{CBF}$ has recently been presented by Winters et al. (2007). For details of recent experimental measurements of the forces associated with cilia beating, see Braiman and Priel (in this volume) and also Teff et al. (2007, 2008).

The beat pattern is asymmetric in time, the recovery stroke and subsequent pause taking several times longer than the effective stroke (Sanderson and Sleigh, 1981). If airway surface liquid behaved as a Newtonian viscous fluid, this time-asymmetry would not have any fluid dynamic function. The possible role of the timeasymmetry in propelling mucus with viscoelasticity or non-linear rheology is a subject that has yet to be investigated theoretically. Sanderson and Sleigh (1981) observed that as the mucous layer deepened, the time-asymmetry of the cilia beat decreased, corresponding to slower cilia movement but engagement of more cilia. The mechanics of this adaptation have not been investigated quantitatively, but we speculate that it would allow the force on individual cilia to be reduced, while the number of cilia contributing propulsive force increases, maintaining MCC. Sanderson and Sleigh (1981) also suggested that the observed 'rest phase' at the end of

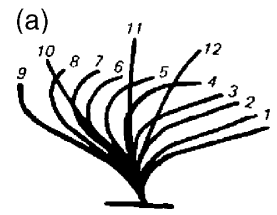

(b)

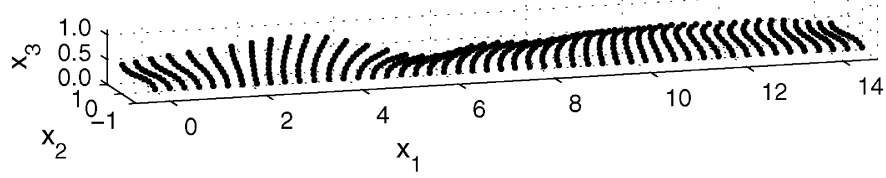

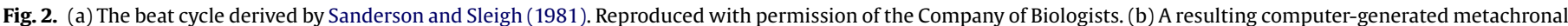
wavelength of cilia, using the analytic representation given by Fulford and Blake (1986b). 
the effective stroke may act to reduce back flow of mucus-again a quantitative analysis of this has not been made.

\subsection{Metachronism}

An important simplifying feature of many mathematical models, as well as a fertile area for other models to understand the emergence of is metachronism. A brief review follows; for further details, see Braiman and Priel (in this volume). Metachronism is a form of coordinated activity whereby the movement of a cilium is consistently out of phase with surrounding cilia. Hence the region of cilia performing the effective stroke propagates, usually in the opposite direction in the case of lung cilia ('antiplectic metachronism'). This is seen most dramatically in swimming microorganisms, which give the appearance of 'the wind blowing over a field of corn'. A computer-generated metachronal wave based on analysis of tracheal cilia is given in Fig. $2 \mathrm{~b}$.

Detailed mathematical analysis of metachronism is given in Gheber and Priel (1990). The wave is split into two components, one parallel to the effective stroke (the 'orthoplectic direction'), the other perpendicular (the 'diaplectic direction'). Mathematical models such as those described in Section 2.2 have typically considered only the effect of metachronism in the orthoplectic direction, assuming synchrony in the diaplectic direction. However, data have shown (Sanderson and Sleigh, 1981) that activity is also propagated in the diaplectic direction, the angle between the effective stroke direction and the wave propagation direction being approximately $135^{\circ}$. Gheber and Priel (1990) derived an expression for the metachronal wavelengths in both directions, based on the assumption that mechanical contact of cilia causes propagation of activity. Their model gave predictions of the angle of propagation and wavelength, the latter comparing very well with experimental studies. It was later shown in frog oesophagus cilia (Gheber and Priel, 1998) that increased viscosity causes the metachronal wave to become relatively more orthoplectic. This modulation was interpreted as being due to a decrease in the relative temporal asymmetry between the effective and recovery strokes. The emergence of metachronism has been the subject of extensive detailed modelling, reviewed in Section 4.2.

\subsection{Airway surface liquid rheology}

Mucus is a gel which exhibits complex rheological properties such as shear-thinning, viscoelasticity, spinnability/cohesiveness and adhesiveness, due to its content of glycosylated mucin polymers (Puchelle et al., 1987). A detailed review of the composition, structure and rheology of mucus may be found in Sleigh et al. (1988), and concise recent reviews of mucus rheology can be found in Majima (2002), King (2004), Bush et al. (2006) and Rubin (2007). Due to varying strain rates, rheology throughout the volume of the mucus may be non-uniform with, for instance, lower viscosity near the zone of ciliary penetration due to shear-thinning. Rheology also varies along the respiratory tree (App et al., 1993). PCL has generally been considered as a watery 'Newtonian fluid' that does not exhibit elasticity or other complex properties, but this has recently been challenged (Boucher, 2007).

Medical research typically has focused on viscosity, which quantifies fluid-like 'thickness', elasticity which quantifies solid-like energy storage, spinnability, which quantifies the ability of mucus to be drawn into a thread, and also surface tension. Verdugo (1990) discusses how rheology is regulated by hydration, and how altered ionic composition of the ASL, characteristic of that observed in $\mathrm{CF}$, can result in poor swelling of secreted mucin granules, and hence excessive viscosity. Feng et al. (1999) showed that adding dextran to mucus reduced viscoelasticity, while increasing MCC.
Recent work has suggested that, in contrast with the classic view of $\mathrm{CF}$ being a disease caused by highly viscous mucus, abnormal rheology may not be the primary cause of the associated illness. Studies have shown that altered biophysical properties of mucus, and possibly MCC dysfunction itself, are secondary to infection in both CF and PCD lung disease (McShane et al., 2004; Grubb et al., 2004; Bush et al., 2006). Viscoelasticity has recently been shown to be an important factor in the formation of bacterial colonies (Matsui et al., 2006).

Theoretical models have generally considered mucus to be Newtonian, neglecting all properties but its viscosity, or alternatively have considered it as a viscoelastic liquid, including energy storage effects. The most complex model so far considered in the literature is a non-linear Maxwell upper convected viscoelastic liquid (Mitran, 2007b). The recent study of Dillon et al. (2007) takes a slightly different approach, modelling mucus as being an immersed elastic network. The relative importance of viscosity and elasticity is measured by the ratio of elastic (storage) and viscous (loss) moduli, $G^{\prime} / G^{\prime \prime}$. Microrheometry data have shown (see, for example, Wolf et al., 1977) that this ratio is close to 1 for tracheal mucus, implying that both viscosity and elasticity are important, although relating experimental measurements to specific biological systems is not necessarily straightforward.

\section{Theoretical models of mucociliary clearance}

\subsection{Motivation for modelling}

While numerous physiological and pathophysiological factors are known to influence MCC and associated diseases, there are often insufficient data to evaluate each of their effects on MCC (Donno et al., 2000), motivating experimental and theoretical modelling studies. Models have been concerned with predicting such features as: the flow profile across the ASL depth, averaged over the beat cycle duration; the effect of altered rheology, CBF, cilia-mucus penetration and other parameters on mean flow; instantaneous flow profiles; the relation between fluid flow and the transport of fluorescently labelled tracer; and the emergence of metachronism.

Studies concerned with the flow velocity profile (see, for example, Fulford and Blake, 1986b) generally predicted small PCL transport in comparison to mucous transport. The ciliated culture study by the Chapel Hill Virtual Lung group (Matsui et al., 1998) led the present authors to re-evaluate these models. A schematic diagram is given in Fig. 3. The cultures, which exhibited rotational transport of mucus, were labelled so that fluorescent 'columns' of surface liquid extended through both the mucous layer and PCL. The labelled regions moved at approximately equal speed in both layers, suggesting approximately equal transport of both mucus and PCL (Fig. 3a). This was in contrast to previous theoretical predictions of very small PCL fluid transport (Fig. 3c), or indeed the flow profile that would be expected from frictional coupling of mucus and PCL alone (Fig. 3b).

The issue of liquid absorption by the epithelium was of great interest, since there were two conflicting theories of the normal ion permeability of the epithelium: does it absorb liquid - as would be required to maintain PCL height if the liquid is significantly transported through the converging geometry of the respiratory tree, or is it impermeable - which allows hypotonic conditions to be maintained in the ASL? The resolution of this question had significant implications for the pathogenesis of CF lung disease.

A series of studies by the Virtual Lung group have firmly established the absorptive role of the epithelium. However, important questions remain: what is the normal level of PCL transport, and what are the physical effects governing MCC? In the following sections we discuss recent progress in understanding the nature of the 


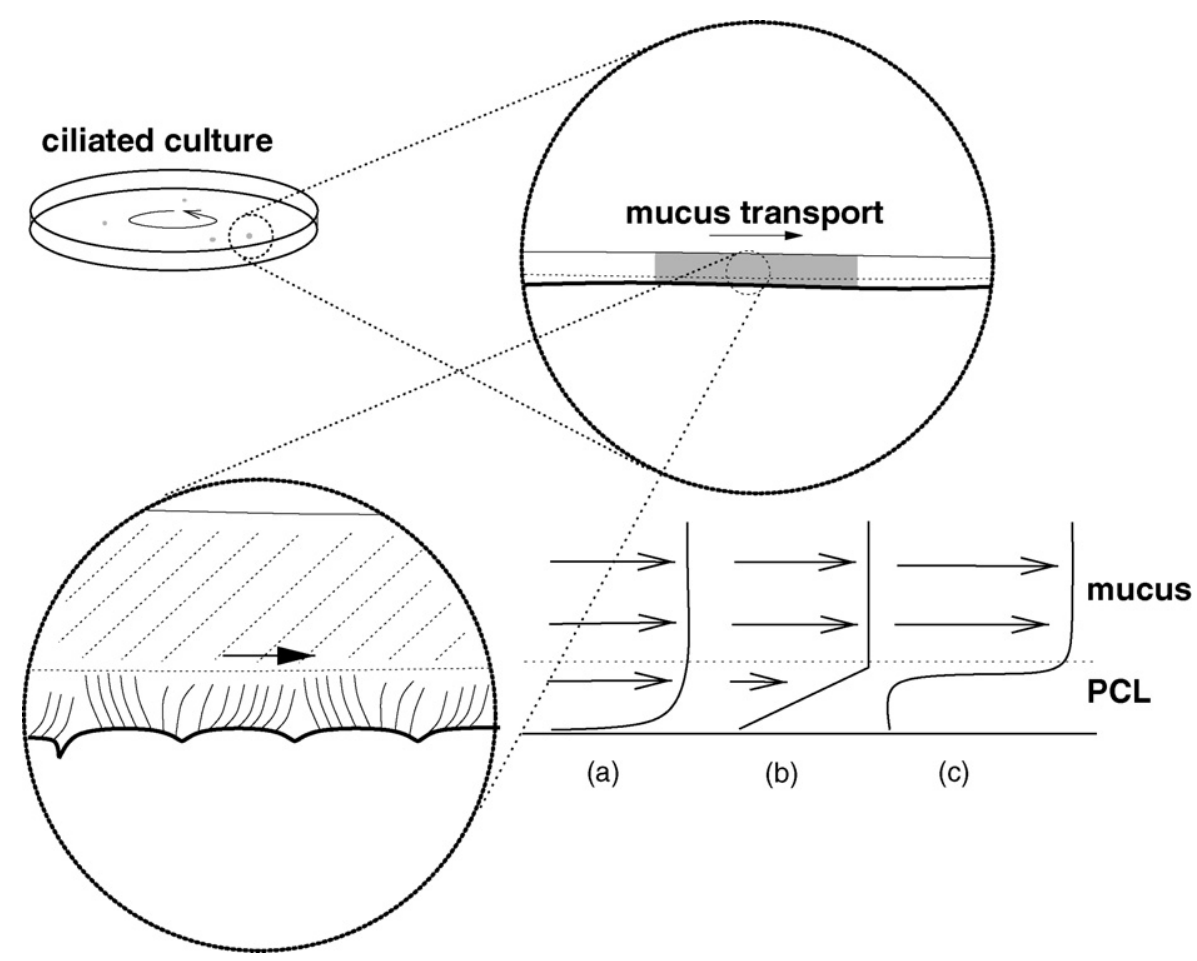

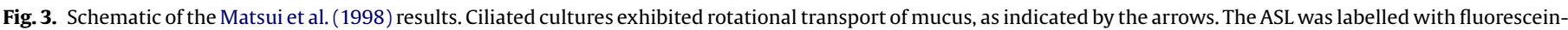

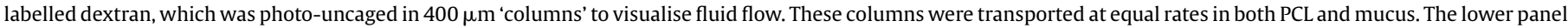

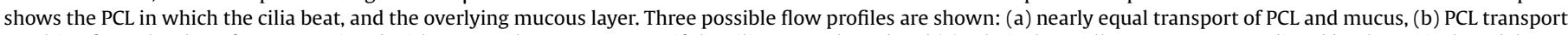
resulting from the shear forces associated with mucous layer movement, if the cilia are neglected and (c) relatively small PCL transport predicted by theoretical models.

mucociliary flow, and the challenges and unresolved questions that present for future research.

\subsection{Review of mucociliary modelling}

Barton and Raynor (1967) developed a mathematical analysis of ciliary propulsion, approximating the cilium by a rigid rod which automatically shortens during the recovery stroke. They used a simplified 'resistance coefficient' approximation to the effect of the cilium on the surrounding fluid, and their model of the cilia motion did not include the experimental findings that were to emerge later. Their work was a forerunner of later 'cilia sublayer' models, and they calculated realistic flow rates. Their characterisation of the cilium as a rigid rod was exploited in the models of Smith et al. (2007c) and Blake and Winet (1980).

The model of Ross (1971) took into account the non-Newtonian nature of the upper mucous layer. Rather than modelling the effect of individual cilia, the mucous layer was propelled by a moving 'wavy wall' representing the cilia tips, and the flow in the PCL was not considered. Mucus was modelled as a non-linear Maxwell fluid, which takes into account viscoelasticity. The resulting system of equations was solved analytically. Mucus flow rates were calculated which were in rough agreement with experimental data, although there are difficulties with the application of wavy-wall modelling to antiplectic cilia motion (Blake, 1972). Nevertheless, modelling of non-linear viscoelasticity represented a significant achievement.

Another important aspect of the study of Ross was the estimation of the capillary number associated with mucociliary flow. This calculation gave an early indication that surface tension effects may be important in governing ASL transport. Analyses which explicitly take into account both surface tension and ciliary transport have yet to be developed, although the phenomenological model described in Section 2.4 suggests that surface tension may have important effects on mucus-cilia coupling.
An alternative 'discrete sublayer' approach exploiting the mathematical technique of slender body theory was developed by Blake (1972), initially for ciliated microorganisms. Due to their slenderness, individual cilia can be modelled by distributing force singularities along their centrelines. These ideas were extended in studies such as Liron and Mochon (1976) and Fulford and Blake (1986b). A detailed review of such models is given in Smith et al. (2007a). Such models have only been applied directly to a one or two-layer Newtonian ASL (Blake, 1973, 1975, 1984; Fulford and Blake, 1986b). The recent model of Smith et al. (2007a) calculates an instantaneous three-dimensional flow field in the PCL, but does so by taking account of viscoelastic flow in the mucous layer indirectly, using a separate phenomenological model.

Mathematically, all of these studies involved representing the flow field by integrals of 'point-force' solutions of the zero-inertia Stokes flow equations. By employing such solutions for flow near to a cell surface (Blake, 1971), or flow due to a slender body near to or indeed straddling a fluid-fluid interface (Fulford and Blake, 1986b), it was possible to calculate approximate time-averaged force distributions on the cilia, and hence time-averaged flow profiles. Certain simplifying assumptions must be made in addition to the assumption of Newtonian rheology: the interaction of the cilium with the time-averaged flow only was calculated, and the interface and surface was assumed not to be subject to surface tension to leading order. Nevertheless, these studies provided considerable insight: it was predicted that the probable quantity of PCL clearance versus mucous clearance was very low, and that mucous transport does not require cilia penetration into the mucous layer, but rather in situations of high ciliary inactivity, penetration is essential for adequate mucous transport.

Keller (1975) developed a phenomenological approach to modelling the sublayer (PCL) in microorganism swimming, referred to as a 'traction layer' model. The action of the cilia was modelled as a spatially continuous volume force. This technique was 


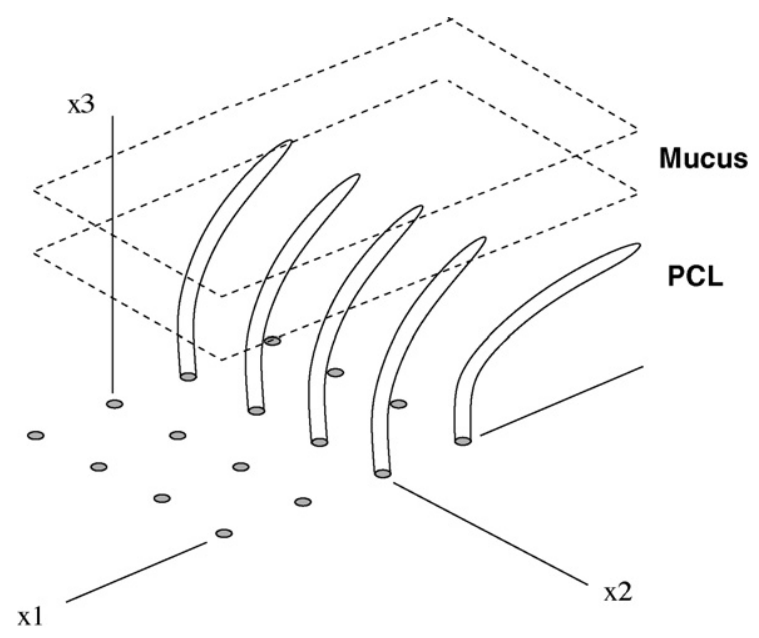

Fig. 4. A representation of a rectangular array of cilia, beating in the $x 1$ direction and coordinated in the $x 2$ direction, as exploited in the 'discrete-cilia' models of Blake, Liron and others described in the text.

applied to ciliated microorganisms where the mucous layer is not present. Blake and Winet (1980) applied the traction layer approach to mucociliary transport in the lung, using the idealised beat pattern of Barton and Raynor (1967). They considered both the PCL and the mucous layers, but modelled mucus as a Newtonian fluid, calculating time-averaged flow only. Cilia sublayer resistance was modelled as an 'active porous medium', building on an earlier paper (Blake, 1977). Their results suggested that slight penetration into the mucous layer by the cilia substantially enhanced transport. They also predicted that mean PCL transport would be very small. The ideas of Blake and Winet (1980) proved a starting point for our recent phenomenological model, described below.

Liron and Rozenson (1983) modelled mucus-cilia interaction in a simplified manner, in order to take account of non-Newtonian effects. Mucus was represented by the linearised Oldroyd B model, valid for small rates of shear. It was assumed that the only forces driving the fluids were a constant pressure gradient and a series of impulses, represented by Dirac delta functions, produced by the cilium tips. After solving the resulting equations by Fourier transforms they concluded that penetration was necessary for transport. Their approach did not however take into account the effect of cilia movement or viscous resistance in the PCL (Fulford and Blake, 1986b). King et al. (1993) formulated a simple analytical model of the mucociliary system, designed to test the effect of mucus viscoelasticity. While their model was based on certain restrictive assumptions, such as zero mean PCL transport, they were able to give insight into the effect of viscoelasticity: it was predited that clearance increases as elastic modulus decreases.

\subsection{Types of model}

There is a hierarchy of fluid dynamical models of different levels of complexity. We shall be concerned only with 'sublayer' models which consider both the PCL and mucous layer, not least because the flow in the PCL is of interest. We distinguish the following types: (1) Volume force, which are phenomenological models representing the cilia with a spatially continuous force distribution, varying in time as the cilia beat. Examples are Keller (1975), Blake and Winet (1980) and Smith et al. (2007c). (2) Discrete-cilia, more precise mathematical models representing the cilia as discrete entities. A schematic of the modelling set-up is shown in Fig. 4. Examples are the models of Blake (1972, 1973) and Fulford and Blake (1986b) described above. For detailed review, see Sleigh et al. (1988). A discrete-cilia model was developed for the case of a single layer fluid to remove the need for time-averaging by Liron and Mochon (1976). This was further developed by Smith et al. (2007a) to obtain three-dimensional flow patterns in the PCL.

Volume force models are generally used to include physical effects that are difficult to take into account in a more precise mathematical model. However, such models are clearly limited in the respect that a number of simplifying approximations must be made in their formulation. Discrete-cilia models are more mathematically rigorous, however the time-dependent modelling has proved difficult to extend to the two-layer mucociliary system in any more than a 'tactical' way (Smith et al., 2007a). Time-independent modelling of, for example, Fulford and Blake (1986b) is limited in that it considers the effect of the cilia only through their interaction with the time-averaged velocity field, however for two decades this work was distinguished as the only model of discrete slender bodies interacting with a two-layer fluid.

Discrete-cilia models can be further subdivided: (1) Prescribed beating, in which the cilia motion is input to the model, as in the work referenced above. (2) Coupled internal mechanics/ fluid-structure interaction, in which the cilia motion emerges from the coupling of a specification of the internal axoneme mechanics and cilium elasticity, and the external viscous forces due to the cilia moving in the fluid. The slender body theory analyses of Gueron, Liron and Levit-Gurevich have modelled the emergence of metachronism of cilia beating in a homogeneous Newtonian liquid. The studies of Brokaw (2002), Brokaw (2005) and Lindemann (2004) were concerned with the complex relationship between internal control of dynein activity and waveform, also for cilia and flagella in homogeneous Newtonian liquids, using relatively simple fluid dynamic modelling. Recently computational models have been applied to the ASL, using the immersed boundary method, and finite volume/finite element solvers. These are discussed in more detail in Section 4.2.

Further refinements can be considered: does the model consider a single Newtonian liquid, two liquids of different viscosity, simple non-Newtonian properties, or more complex rheology? In the case of coupled internal mechanics/fluid models, the internal mechanics may be specified in a very simple manner, for example, curvature control, or instead a discrete internal structure may be considered. The mucus-PCL interface must also be considered: this is only tracked 'explicitly' as a coupled part of the calculation by very recent computational models. Finally, we distinguish between models which are two-dimensional (Smith et al., 2007c; Dillon et al., 2007), three-dimensional but which lead to two-dimensional results only (Fulford and Blake, 1986b), three-dimensional in the near-field and two-dimensional in the far-field (Smith et al., 2007a), or fully three-dimensional (Mitran, 2007b). The specific choice has largely been dictated by computational cost, but the latter study shows that fully three-dimensional simulation is now possible.

As models increase in complexity, there are great technical challenges to be overcome in order to extract solutions or useful information. For this reason, simple phenomenological models are still useful in order to gain important insights, as discussed in the following section.

\subsection{Phenomenological modelling}

The mucociliary system is challenging to model without pursuing state-of-the-art computational techniques. The discrete-cilia approach described above employs slender body theory, avoiding the high computational complexity of immersed boundary or finite volume/finite element methods. This enables computationally inexpensive calculation of three-dimensional, time-dependent solutions. However, the mucociliary system has a number of features that make its application problematic. 
Slender body theory is typically used in situations where cilia project from an approximately flat surface, with no other boundaries or fluid interfaces nearby. In such situations, it can be used in a straightforward manner: see, for example, the work of Blake (1972) on microorganism swimming, the coupled cilia/fluid modelling of Liron, Gueron and Levit-Gurevich reviewed below, and the embryonic cilia modelling of Smith et al. (2007a). However, as discussed above, the mucociliary system presents the complication that cilia beat in a low viscosity layer with an overlying high viscosity nonNewtonian layer. Slender body theory is not strictly valid in such a system. Assuming 'deep' penetration into the mucous layer, Fulford and Blake (1986a) derived a slender body theory for a cilium straddling a two-fluid interface, however it was necessary to make the assumption the overlying layer was Newtonian and surface tension was assumed to be a subleading effect. As shown by Ross (1971) this may be a dominant effect in the absence of airway surfactants. Slender body models also lose accuracy in the situation that the cilia are very closely spaced (Smith et al., 2007a).

For these reasons, we pursued a phenomenological modelling approach, taking into account the most important features of the cilia propulsion, sublayer 'active porous medium' resistance, metachronism, and a simple model of mucous layer viscoelasticity. The SEM data of Sanderson and Sleigh (1981) showed that, even in samples in which the epithelium was far from being flat, the mucous layer surface and interface did not show large deformations. An initial model (Smith, 2006) assumed negligible surface tension. With scaling arguments, this assumption led to the conclusion that the internal pressure of the liquid layer is constant throughout the metachronal wave. This model predicted significant vertical components to the fluid motion, causing sufficiently large oscillations in the layers to invalidate the model. The model also predicted that for low viscosity mucus, propulsion was almost abolished.

Consequently, we developed a refined model (Smith, 2006; Smith et al., 2007c), which was based on the assumption that surface and interface tension effects were sufficiently strong that the layer could be assumed flat at all times. Mucus elasticity may also have an important role, as shown by the results of Dillon et al. (2007) discussed in Section 4.2. The assumption of a flat surface is supported by calculations of the capillary number associated with the ASL, which shows that surface tension effects are several times larger in magnitude than viscous effects. In the presence of airway surfactant, particularly in the distal airways, this calculation

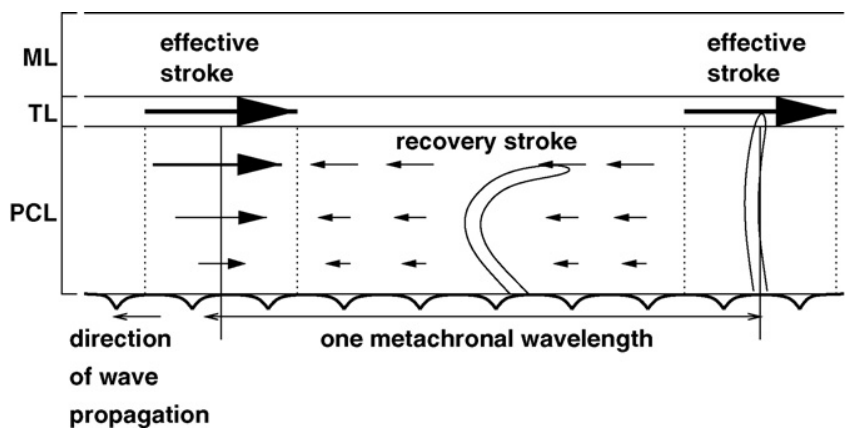

Fig. 5. A schematic representation of the phenomenological model of Smith et al. (2007c). The ASL is divided into three regions: the PCL, the 'traction layer' (TL) in which the cilium tips interact with the mucous layer, and the mucous layer above the cilia tips (ML). The upper surface of the ML, and the interface of the PCL and TL are constrained to be flat. The volume forces replacing the effective and recovery strokes are indicated by arrows.

would have to be modified. Values of the surface tension of sputum and culture surface liquid have been given by Albers et al. (1996) as approximately $0.08 \mathrm{~N} \mathrm{~m}^{-1}$ and Tarran et al. (2001) $0.03 \mathrm{~N} \mathrm{~m}^{-1}$, respectively.

A schematic of the model is shown in Fig. 5, and example results are shown in Fig. 6. By reintroducing surface tension, horizontal pressure gradients in the mucus and PCL were restored. These pressure gradients enforce conservation of mass while the layer height remains constant. In the absence of the pressure gradient, calculations predict that there is a positive horizontal velocity gradient to the left of the effective stroke and a negative horizontal velocity gradient to the right. By conservation of mass, this leads to downward flow to the left of the effective stroke and upward flow to the right of the effective stroke. The large downward and upward flow each side of the effective stroke would change the layer heights by an unphysical amount.

Fluid pressure is lower to the left of the effective stroke than to the right, causing a backflow near the epithelium during the effective stroke, as shown in Fig. 6a. This also results in a steady forwards flow of mucus during the recovery stroke, despite the fact that the cilia are dragging fluid backwards in the PCL. Fig. $6 \mathrm{~b}$ and $\mathrm{c}$ shows that the instantaneous flow profiles may be quite complicated, and that the instantaneous velocity during the effective stroke is an order of magnitude larger than the time-averaged flow. The time-averaged
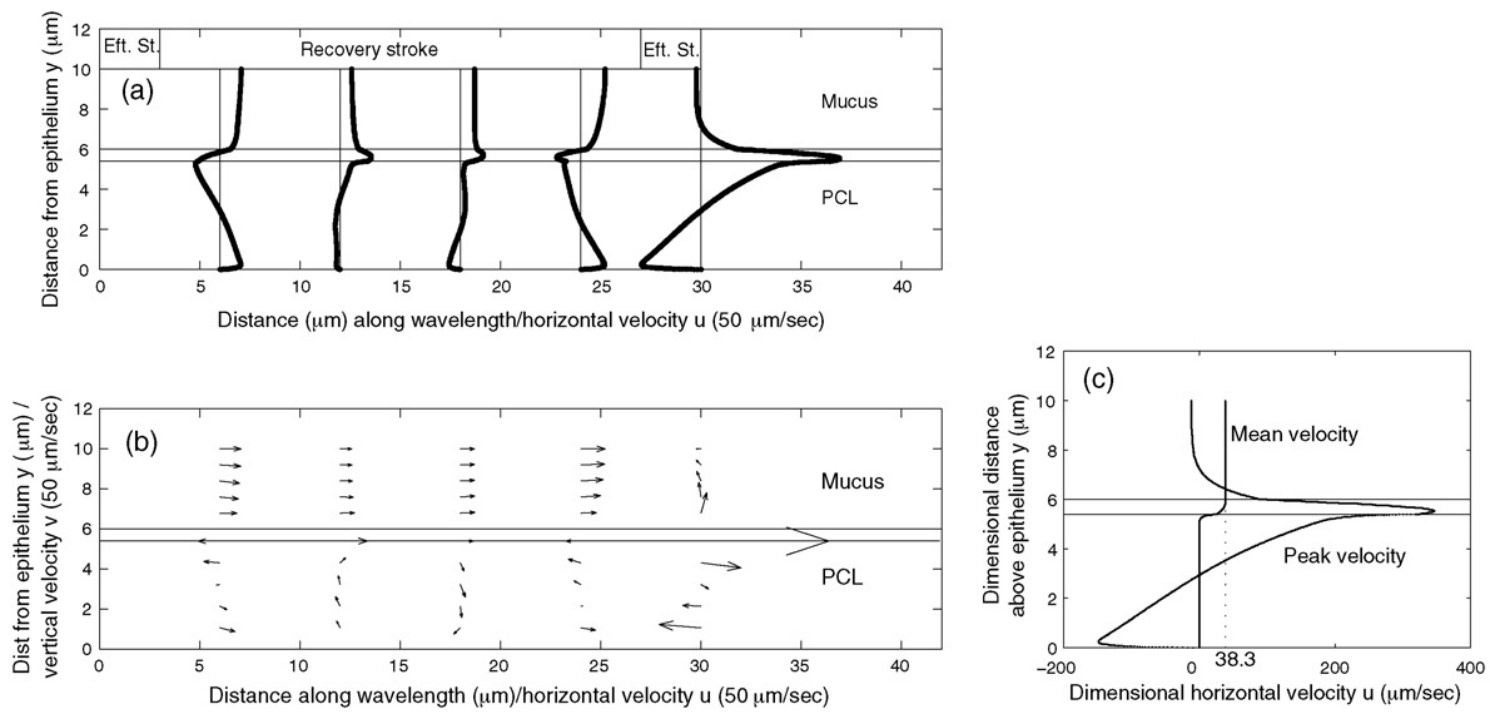

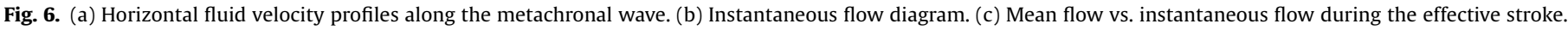
Adapted from Smith et al. (2007c), with kind permission of Springer Science and Business Media. 
flow profile in the PCL is very small, in agreement with previous models. The mean mucus velocity is approximately $40 \mu \mathrm{m} / \mathrm{s}$. The most dramatic effect of the pressure gradient is to couple the cilia and mucus efficiently, even in the absence of high mucus viscosity. Because the pressure results in an increased cilium/liquid relative velocity, the force imparted to the mucus by the beating cilia is increased. An alternate perspective is that the flat interface may be brought about by mucus elasticity, as shown by the immersed boundary simulation results of Dillon et al. (2007).

\section{Links to experiment and clinical findings}

\subsection{Tracer transport modelling}

To investigate which time-averaged flow profiles could result in the observed equal transport of tracer in both mucus and PCL, the present authors (Smith et al., 2007b) formulated and solved a twodimensional advection-diffusion model of the transport of a tracer, with steady and oscillatory profiles calculated with the volumeforce modelling described above. This model took into account the three main effects governing tracer transport: time-averaged advection, due to the mean flow, unsteady advection, due to ciliadriven oscillations, and diffusion across the thin layer, which is enhanced by the shearing associated with the flow profile.

Simulations with a steady flow profile with very small PCL transport do not result in equal transport between the layers, as shown in the example results given in Fig. 7a and b. However, once physically reasonable oscillations in the ASL are taken into account, as shown in Fig. 6, the advection-diffusion model predicts very similar transport of tracer in the two compartments, differing by only $18 \%$ (Fig. 7c and d) despite the fact that the time-averaged transport of liquid in the PCL is relatively very small. When fluid transport in the upper part of the PCL, due to the cilia bending down below the interface during the recovery stroke, was included in the model, the predicted difference in tracer transport was reduced to $9 \%$. Hence very low PCL transport, predicted by the mechanical model on the basis that the cilia resist fluid flow like a porous medium, is not inconsistent with the experimental results on tracer transport. It is nevertheless possible that taking into account osmotic fluxes, or by modelling the PCL and mucous layers in a more sophisticated manner, conclusions regarding mean PCL fluid transport may be different.

\subsection{Robustness of the mucociliary system}

The phenomenological model of Smith et al. (2007c) described above predicted that provided that the flat interface and surface were maintained, clearance is not significantly reduced by any variations in mucus viscosity, elasticity or layer height. The only parameter that did have a significant effect was CBF - reducing this caused an approximately proportional decrease in transport speed. This finding paralleled experimental findings regarding adrenergic blockers and agonists. It remains to determine the mechanics of how MCC is reduced by allergic challenge, in view of the data (Maurer et al., 1982) that CBF increases in such conditions.

'Watery mucus' having similar properties to the PCL, was predicted to be transported around 50\% faster than 'normal mucus', provided that a flat interface was maintained between the two layers. It was suggested that this may be the mechanism for the large increase in ASL transport observed in pseudohypoaldosteronism-a condition in which liquid absorption by the epithelium is greatly impaired.

Hence this phenomenological model provides evidence that pressure gradients may ensure robustness of MCC to changing conditions, such as the changes that occur along the airway as the ASL deepens, and in disease states which are not sufficiently severe as to disrupt the normal ASL structure. A limitation of this work is that $\mathrm{CBF}$ and beat pattern were prescribed, whereas in the real system these parameters will be modulated by mechanical and other effects, including viscosity (Gheber and Priel, 1998), the presence of the mucous layer (Matsui et al., 1998), shear stress due to breathing (see Button and Boucher, in this volume) and coughing.
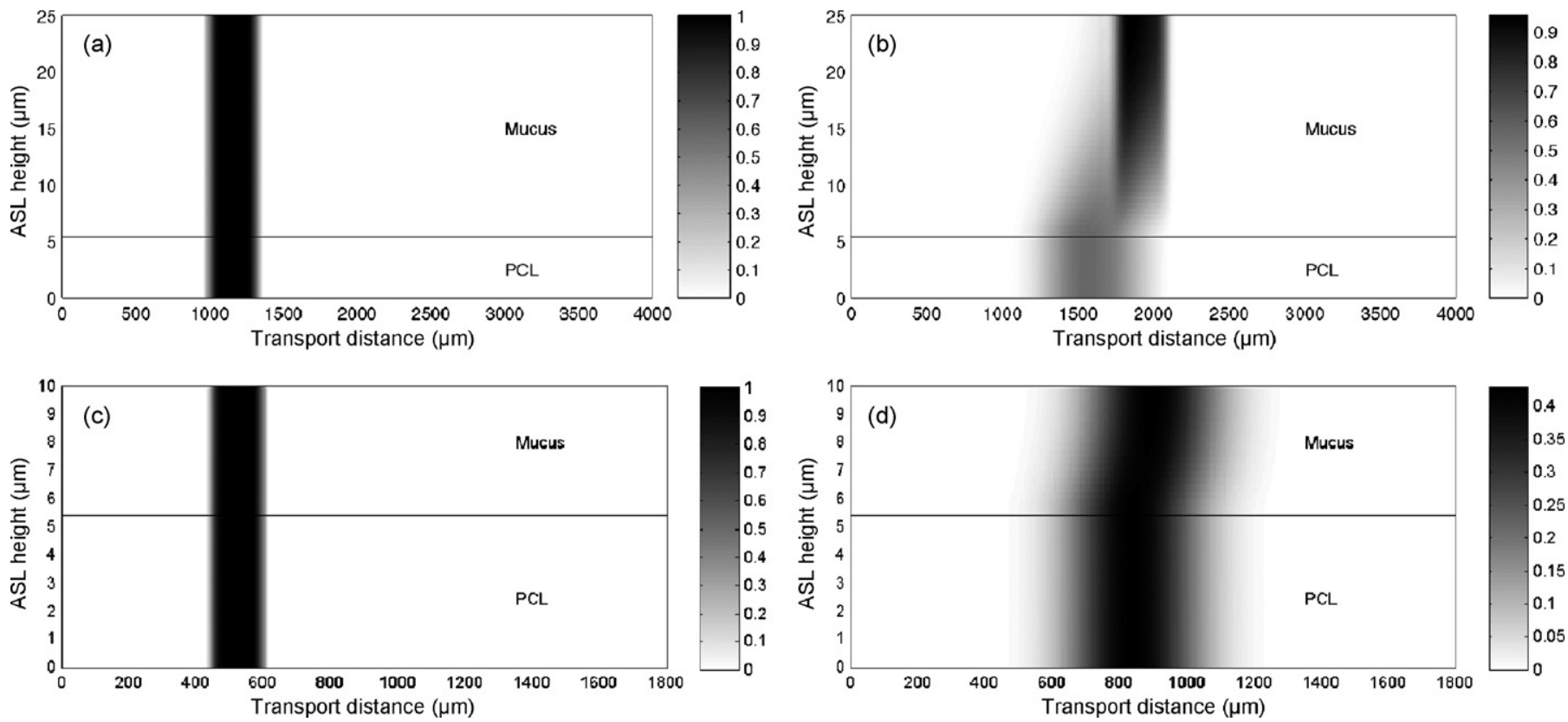

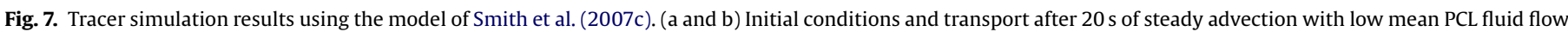

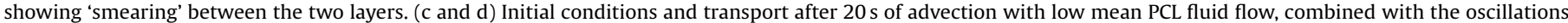
shown in Fig. 6. Tracer is transported with nearly equal velocity across the two layers. 


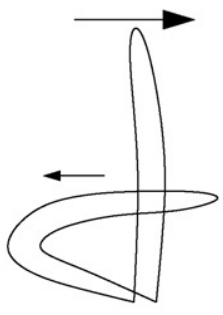

(a) orientation effect

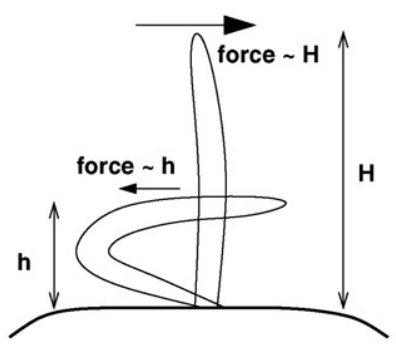

(b) wall effect

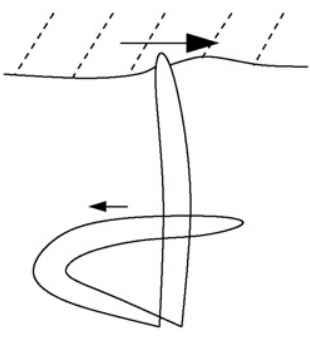

(c) viscosity effect

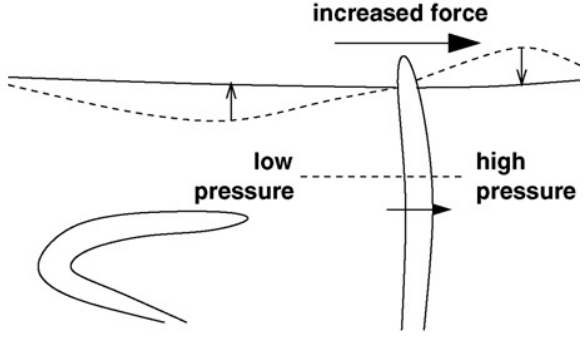

(d) pressure gradient effect

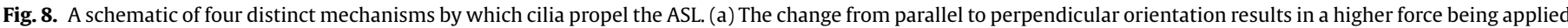

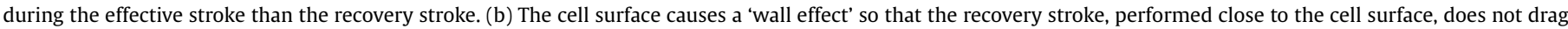

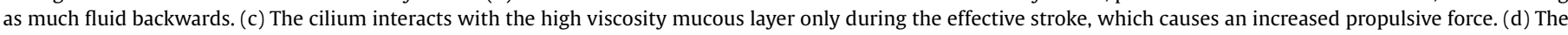
flatness of the interface and surface cause a pressure gradient which increases the forces on the cilium, amplifying the propulsive effect.

\section{Overview and future directions}

\subsection{Cilia transport mechanisms}

Blake (2001) summarised the mechanisms by which cilia can transport liquid, which we illustrate in Fig. 8a-c and briefly review below. For water-propelling cilia, there are: (1) The orientation effect-a slender body having a predominant motion normal to its centreline will exert almost twice the force as for a tangential mode of motion at the same velocity. In cilia this is exploited by the body performing a motion which is approximately normal to the fluid during the effective stroke, and is relatively more tangential during the recovery stroke. (2) The wall effect-the interaction of the cilium with the ASL is 'shielded', reducing the force applied, by a greater degree during the recovery stroke than the effective stroke. For cilia in the lung, there is additionally (3) The viscosity effect-by which cilia interact with high viscosity mucus only during the effective stroke, increasing the force associated with the propulsive action. A principle finding of the model of Fulford and Blake (1986b) was that '...in the mucous layer the extremely high viscosity requires only a small number of cilia per wavelength to penetrate the mucous layer to propel the mucus at near the tip speed of the cilia.'

Since mucus-propelling cilia are relatively short compared with water-propelling cilia, and there is hence less shape and height asymmetry between effective and recovery strokes, this led to the assumption in the phenomenological model of Smith et al. (2007c) that the viscosity effect was the primary mechanism by which transport is produced. As discussed above, introducing pressure gradients associated with a flat interface and surface of the two-layer system, led to efficient transport even when the viscosity of the mucous layer was reduced. Hence a fourth mechanism by which cilia can produce effective transport was suggested: (4) The pressure gradient effect-the mucous layer interface and surface remain relatively flat, despite the cilia movement. Associated pressure gradients occur which are negative during the effective stroke and positive during the recovery stroke. This amplifies the forces exerted by the cilium on the liquid, resulting in increased transport, as illustrated in Fig. 8d.

This latter effect may be tested using the modelling framework presented by Dillon et al. (2007). Osmotic pressure gradients may be present in the ASL, due to the movement of ions and associated movement of water across the epithelial cell membrane. Such pressure gradients are likely to affect cilia-fluid forces and hence ASL velocity in a similar way, and may act over larger distances than those associated with the metachronal wavelength $(30 \mu \mathrm{m})$.

\subsection{Fluid-structure interaction modelling}

To understand how cilia beating and fluid transport emerge as consequences of fluid mechanics, rheology and the internal mechanics of the cilia, it is necessary to solve a 'fluid-structure interaction' problem. Early pioneering studies using a simplified form of slender body theory, together with increasingly detailed models of internal axoneme activity, included Machin (1958), Brokaw (1972) and Hines and Blum (1978). A more accurate model of the fluid mechanics was introduced by Gueron and Liron (1992), which allowed the modelling of lines of beating cilia, immersed in a homogeneous Newtonian liquid, their bases attached to a solid surface. A series of publications followed exploring the energetic advantages of metachronal coordination, adaptations in CBF to fluid viscosity, the movement of energy substrate along cilia from the cell, and modelling of three-dimensional bending and cilium torsion (Gueron and Liron, 1993, 1998; Gueron and Levit-Gurevich, 1999, 2001) Other detailed modelling of the axoneme and associated computer simulation were presented recently by Lindemann (2004) and Brokaw (2002, 2005), the latter paper investigating how embryonic nodal cilia establish 'whirling' motility by considering internal asymmetry of the microtubule system. Nevertheless, as discussed above the mucociliary system has features that make further generalisation of this modelling problematic.

Very recently two important studies have appeared which have applied sophisticated computational techniques to the problem, which promise to allow simulation of the mucociliary system with considerable flexibility regarding internal cilium mechanics and mucus rheology. Dillon et al. (2007) used the immersed boundary method to simulate two-dimensional flow due to three cilia, with a discrete model of the internal elastic and force-generating structures of the cilia. The mucous layer was simulated using a relatively thin elastic layer immersed in the fluid, a short distance above the cilia tips. Their initial results showed that elastic properties of the mucous layer could prevent unphysical deformation of the mucus-PCL interface discussed above. Hence considering the mucous layer as an elastic solid, rather than a viscoelastic liquid with surface tension the dominant effect, also led to physically reasonable results (Fig. 9). Moreover, the cilia were able to propel marker particles without the need to engage with the mucous layer, as observed experimentally by Winet (1987). While this study considered a small number of cilia and twodimensional flow, the framework provides a promising avenue for future research.

Mitran (2007b) recently presented impressive initial computations, using the finite element method for the simulation of the microtubule internal structures of multiple cilia, and the finitevolume method for the fluid flow equations. The PCL was modelled as Newtonian, the mucous layer as an upper-convected Maxwell fluid. This approach allowed for the first time the simulation of three-dimensional flow due to a line of 256 cilia, with simulation of internal mechanics. Metachronism was found to emerge in the simulated system. Unpublished data (Mitran, 2007a) describe the 


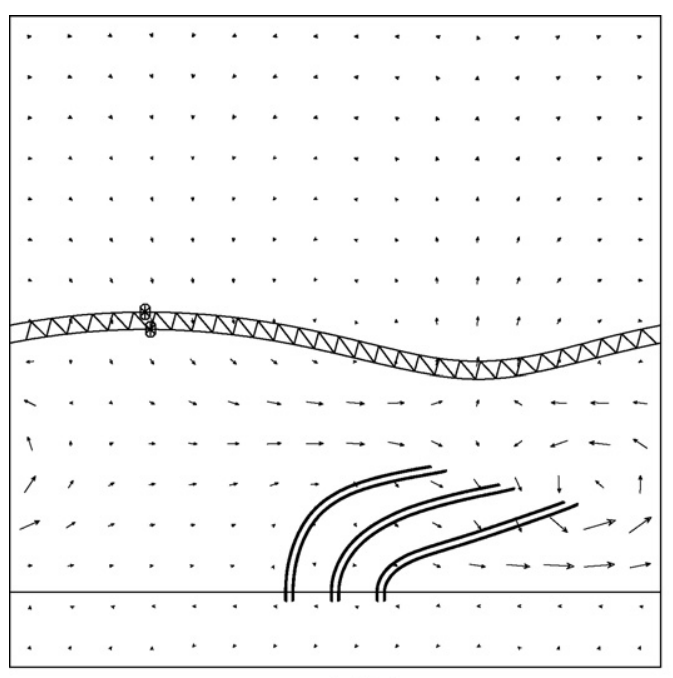

$t=0.004 \mathrm{~s}$

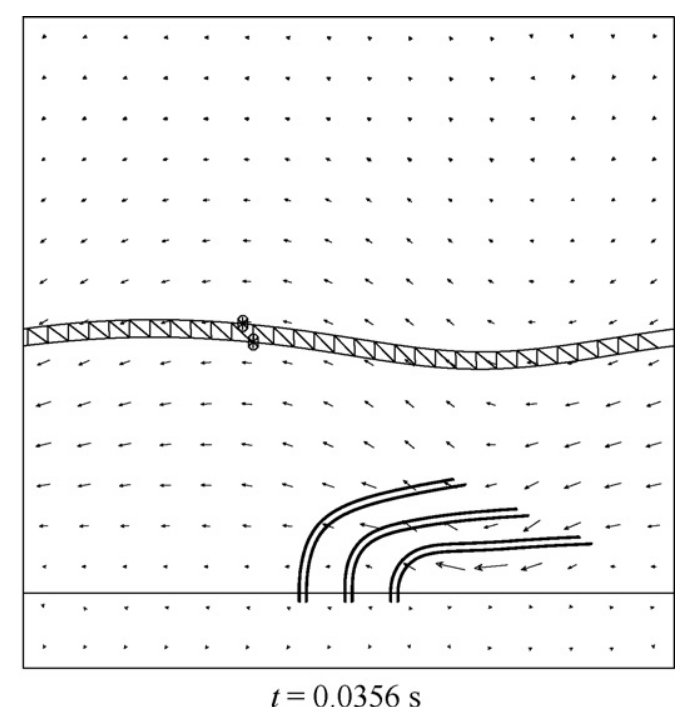

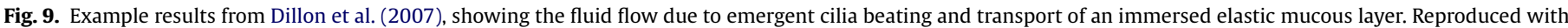
permission of Blackwell Publishing.

simulation of a three-dimensional array of 16,384 cilia, including for the first time a prediction of the emergence of three-dimensional metachronal activity.

\subsection{Limitations of current models}

There remain a number of areas where presently published models are limited, as well as a significant number of questions regarding MCC that have not yet been addressed. While the recent phenomenological modelling of Smith et al. (2007c) has provided some insights, important questions remain. It is unclear whether cilia actually resist the flow of liquid in the same manner as a porous medium. It is also difficult to extend the framework to take into account more complex fluid rheology. Finally, the 'volume force' model of the cilia action does not allow for analysis of subtle interaction between the fluid and the cilia beat generation. Nevertheless, this study suggests a number of insights regarding the effects of rheology, surface tension and transport of fluorescent tracer that may prove useful for future study. Slender body models such as that of Fulford and Blake (1986b) have also proved very successful, but as discussed above, have certain limitations when applied to the two-layer mucociliary system.

The computational frameworks described in Section 4.2 promise to provide a platform to test all of the predictions of previous models, in addition to including detailed specification of fluid rheology and cilium internal mechanics. An important question to address is how precisely the interface and surface boundary conditions will be modelled. This requires both empirical measurements of properties such as surface tension, and additionally decisions regarding the modelling of a Newtonian/viscoelastic interface. The latest generation of models that include cilium internal mechanics do not yet have a definitive specification for how dynein arms initiate or propagate bending. Lindemann (2004) describes in detail the 'geometric clutch' hypothesis for the control of flagellar bending by dynein activity, showing computed beat patterns calculated with a simple model of the fluid flow and fluid-structure interaction. Brokaw $(2002,2005)$ give simulation results showing three-dimensional beating due to curvature-controlled dynein activity, and sliding-velocity controlled dynein activity, respectively. The former analysis was concerned with flagellar movement, but the internal mechanics considered transfer directly to the movement of cilia.
Perhaps the most difficult limitation to overcome is the fact that all present models are based on continuum mechanics. Noncontinuum effects, for example, nano-scale interactions between the cilium tip and mucus polymer molecules (Blake, 2001), which have been speculated to be an important feature of cilia-mucus coupling, cannot be explicitly included within a continuum framework. Modelling such interaction presents a significant challenge.

\subsection{Future work}

Future work may develop through the refinement of existing models by including increasingly more accurate rheology, fluid mechanics and cilium internal mechanics. This will involve the integration of more accurate biological data with the new generation of computational models.

An example problem is determining the possible role of PCL viscoelasticity in normal functioning and disease. Knowles and Boucher (2002) noted that increased liquid volume in PHA may result in reduced PCL viscoelasticity, which may increase clearance. We have preliminary modelling data using an extension of slender body theory that suggests that viscoelastic effects may substantially reduce both the forces on cilia - enabling continued beating at high viscosity - but also reduce clearance. An other example is determining the effect of loss of ciliated epithelium, including lesions and intercellular space edema, characteristic of asthma (Donno et al., 2000). Fulford and Blake (1986b) provided insight into the effect of reduced ciliary activity, and the compensating role of cilium-mucus interaction. However, the effect of large deciliated regions is not yet understood theoretically. It may be that the rheological properties of mucus are important in preserving flow over such regions.

Future modelling will also progress through the inclusion of progressively more of the multiscale biological system, leading eventually to models of all or part of the organ, as is being pursued by the Chapel Hill group. We conclude by describing some important examples of the wider biological system.

On the sub-micron scale, models for the coupled beating and internal mechanics of cilia may in future be developed to take into account signalling to and from the epithelial cell, and other aspects of the molecular biology. For review see Satir and Christensen (2007) and for recent experimental results see Winters et al. (2007). On the $1-10 \mu \mathrm{m}$ scale, the establishment and dysfunction of the mucus-PCL bilayer is not fully understood, and molecular-level 
models remain to be developed which consider the possible role of cilia, interacting with mucin molecules, in producing this system, and the breakdown of the system in disease states. The glycocalyx (Knowles and Boucher, 2002) has not been included in any theoretical models, and may have important effects on fluid transport. A model of endothelial glycocalyx has been given by Weinbaum et al. (2003). Additionally, modelling of the secretion of mucin granules, their hydration, and the effect of ion concentrations and cilia movement, coupled back to the effect of mucus hydration on rheology and hence ASL transport may give further insight.

On longer length scales, osmotic fluxes through the epithelium will have at the very least transient effects on the fluid flow in the PCL in the in vivo situation. This may increase the flow in the PCL significantly, provided the pressure gradients created are sufficiently large. On the millimetre to centimetre lengthscale, the effect of the bifurcating structure of the airways has not been taken into account in any mathematical models. The fluid dynamics of airway surface liquid at and near to these bifurcations may be considered, and variations in the fluid transport along airways between bifurcations may also be of interest, particularly in determining how the ASL is regulated, possibly by osmosis or by mucus hydration, as the liquid depth increases due to a bifurcation. Models may be developed of the global balance of fluid and salt, which could then be integrated with models of how ion channels are regulated by nucleotides and other signals, and how in turn these signals may be regulated by shear stress. This would enable an understanding of the wider system that regulates PCL depth and the assessment of parameters governing its normal modulation. Finally, models of MCC have not been coupled to models for the formation and breakup of clinically important mucus plugs. The effect of cilia beating on the movement on such a plug does not appear to have been studied.

\section{Acknowledgement}

DJS acknowledges a Medical Research Council Special Training Fellowship in Computational Biology.

\section{References}

Albers, G.M., Tomkiewicz, R.P., May, M.K., Ramirez, O.E., Rubin, B.K., 1996. Ring distraction technique for measuring surface tension of sputum: relationship to sputum clearability. J. Appl. Physiol. 81 (6), 2690-2695.

App, J., Zayas, J.G., King, M., 1993. Rheology of mucus and transepithelial potential difference: small airways versus trachea. Eur. Respir. J. 6, 67-75.

Barton, C., Raynor, S., 1967. Analytical investigation of cilia induced mucous flow. Bull. Math. Biophys. 29, 419-428.

Blake, J.R., 1971. A note on the image system for a stokeslet in a no slip boundary. Proc. Camb. Philos. Soc. 70, 303-310.

Blake, J.R., 1972. A model for the micro-structure in ciliated organisms. J. Fluid Mech. $55,1-23$

Blake, J.R., 1973. Mucus flows. Math. Biosci. 17, 301-313.

Blake, J.R., 1975. On the movement of mucus in the lung. J. Biomech. 8, 179-190.

Blake, J.R., 1977. An active porous medium model for ciliary propulsion. J. Theor. Biol. 64, 697-701.

Blake, J.R., 1984. Mechanics of muco-ciliary transport. IMA J. Appl. Math. 32, 69-87.

Blake, J.R., 2001. Fluid mechanics of ciliary propulsion. In: Fauci, L.J., Gueron, S. (Eds.) Computational Modeling in Biological Fluid Dynamics. Springer, pp. 1-52.

Blake, J.R., Winet, H., 1980. On the mechanics of muco-ciliary transport. Biorheology $17,125-134$

Boucher, R.C., 2007. Airway surface dehydration in cystic fibrosis: pathogenesis and therapy. Ann. Rev. Med. 58, 157-170.

Brokaw, C.J., 1972. Computer simulation of flagellar movement. I. Demonstration of stable bend propagation and bend initiation by a sliding filament model. Biophys. J. 12, 564-586.

Brokaw, C.J., 2002. Computer simulation of flagellar movement. VII. Coordination of dynein by local curvature control can generate helical bending waves. Cell Motil. Cytoskel. 53, 102-124.

Brokaw, C.J., 2005. Computer simulation of flagellar movement. IX. Oscillation and symmetry breaking in a model for short flagella and nodal cilia. Cell Motil. Cytoskel. 60, 35-47.
Bush, A., Payne, D., Pike, S., Jenkins, G., Henke, M.O., Rubin, B.K., 2006. Mucus properties in children with primary ciliary dyskinesia: comparison with cystic fibrosis. Chest 129 (1), 118-123.

Chilvers, M.A., O'Callaghan, C., 2000. Analysis of ciliary beat pattern and beat frequency using digital high speed imaging: comparison with the photomultiplier and photodiode methods. Thorax 55, 314-317.

Chilvers, M.A., Rutman, A., O'Callaghan, C., 2003. Ciliary beat pattern is associated with specific ultrastructural defects in primary ciliary dyskinesia. J. Allergy Clin. Immunol. 112 (3), 518-524.

Davis, C.W., Dickey, B.F., 2008. Regulated airway goblet cell mucin secretion. Ann. Rev. Physiol., 70.

Dillon, R.H., Fauci, L.J., Omoto, C., Yang, X., 2007. Fluid dynamic models of flagellar and ciliary beating. Ann. N. Y. Acad. Sci. 1101, 494-505.

Donno, M.D., Bittesnich, D., Chetta, A., Olivieri, D., Lopez-Vidriero, M.T., 2000. The effect of inflammation on mucociliary clearance in asthma. Chest 118 , $1142-1149$.

Feng, W., Nakamura, S., Sudo, E., Lee, M.M., Shao, A., King, M., 1999. Effects of dextran on mucociliary velocity in dogs in vivo. Pulm. Pharm. Theraput. 12 (1), 3541.

Fulford, G.R., Blake, J.R., 1986a. Force distribution along a slender body straddling an interface. J. Aust. Math. Soc., Ser. B, Appl. Math. 27 (3), 295-315.

Fulford, G.R., Blake, J.R., 1986b. Muco-ciliary transport in the lung. J. Theor. Biol. 121, 381-402.

Gheber, L., Priel, Z., 1990. On metachronism in ciliary systems: a model describing the dependence of the metachronal wave properties on the intrinsic ciliary parameters. Cell Motil. Cytoskel. 16, 167-181.

Gheber, L., Priel, Z., 1998. Effect of viscosity on metachrony in mucus propelling cilia. Cell Motil. Cytoskel. 39, 9-20.

Groneberg, D.A., Wagner, U., Chung, K.F., 2004. Mucus and fatal asthma. Am. J. Med. 116, 66-67.

Grubb, B.R., Jones, J.H., Boucher, R.C., 2004. Mucociliary transport determined by in vivo microdialysis in the airways of normal and $\mathrm{cf}$ mice. Am. J. Physiol. Lung Cell. Mol. Physiol. 286, L588-L595.

Gueron, S., Levit-Gurevich, K., 1999. Energetic considerations of ciliary beating and the advantage of metachronal coordination. Proc. Natl. Acad. Sci. U.S.A. 96 (22), $12240-12245$

Gueron, S., Levit-Gurevich, K., 2001. A three-dimensional model for ciliary motion based on the internal 9+2 structure. Proc. R. Soc. Lond. B 268, 599-607.

Gueron, S., Liron, N., 1992. Ciliary motion modeling, and dynamic multicilia interactions. Biophys. J. 63, 1045-1058.

Gueron, S., Liron, N., 1993. Simulations of three-dimensional ciliary beats and cilia interactions. Biophys. J. 65, 499-507.

Gueron, S., Liron, N., 1998. Computations of the internal forces in cilia: application to ciliary motion, the effects of viscosity, and cilia interactions. Biophys. J. 74, 1658-1676.

Hines, M., Blum, J.J., 1978. Bend propagation in flagella. I. Derivation of equations of motion and their simulation. Biophys. J. 23 (2), 41-57.

Keller, S.R., 1975. Fluid mechanical investigations of ciliary propulsion. Ph.D. Thesis. California Institute of Technology.

King, M., 2004. Physiology of mucus clearance. Paed. Respir. Rev. 7S, S212-S214.

King, M., Agarwal, M., Shukla, J.B., 1993. A planar model for muco-ciliary transport: effect of mucus viscoelasticity. Biorheology 30, 49-61.

Knowles, M., Boucher, R.C., 2002. Mucus clearance as a primary innate defense mechanism for mammalian airways. J. Clin. Invest. 109 (5), 571-577.

Lindemann, C., 2004. Testing the geometric clutch hypothesis. Biol. Cell 96, 681690.

Liron, N., Mochon, S., 1976. The discrete-cilia approach to propulsion of ciliated microorganisms. J. Fluid Mech. 75, 593-607.

Liron, N., Rozenson, M., 1983. Muco-ciliary transport. J. Submicrosc. Cytol. 15 (1), 317-321.

Machin, K.E., 1958. Wave propagation along flagella. J. Exp. Biol. 35 (4), 796-806.

Majima, Y., 2002. Mucoactive medications and airway disease. Paed. Respir. Rev. 3, 104-109.

Marino, M.R., Aiello, E., 1982. Cinemicrographic analysis of beat dynamics of human respiratory cilia. Cell Motil. Supp. 1, 35-39.

Maurer, D.R., Sielczak, M., Oliver Jr., W., Abraham, W.M., Wanner, A., 1982. Role of ciliary motility in acute allergic mucociliary dysfunction. J. Appl. Physiol. 52, 10181023.

Matsui, H., Randell, S.H., Peretti, S.W., Davis, C.W., Boucher, R.C., 1998. Coordinated clearance of periciliary liquid and mucus from airway surfaces. J. Clin. Invest. 102 (6), 1125-1131.

Matsui, H., Wagner, V.E., Hill, D.B., Schwab, U.E., Rogers, T.D., Button, B., Taylor R.M., Superfine, R., Rubenstein, M., Iglewski, B.H., Boucher, R.C., 2006. A physical linkage between cystic fibrosis airway surface dehydration and pseudomonas aeruginosa biofilms. Proc. Natl. Acad. Sci. U.S.A. 103 (48), 18131-18136.

McShane, D., Davies, J.C., Wodehouse, T., Bush, A., Geddes, D., Alton, E.W.F.W., 2004. Normal nasal mucociliary clearance in CF children: evidence against a CFTRrelated defect. Eur. Respir. J. 24, 95-100.

Mitran, S., 2007a. Computation of advection and diffusion of a passive tracer in a prescribed velocity field. http://coanda.amath.unc.edu/mitran/Cilia.

Mitran, S., 2007b. Metachronal wave formation in a model of pulmonary cilia. Comput. Struct. 85 (1114), 763-774.

Puchelle, E., Zahm, J.M., Quemada, D., 1987. Rheological properties controlling mucociliary frequency and respiratory mucus transport. Biorheology 24 557-563. 
Rogers, D.F., 2004. Airway mucus hypersecretion in asthma: an undervalued pathology? Curr. Opin. Pharm. 4, 241-250.

Rogers, D.F., 2005. Mucociliary disfunction in COPD: effect of current pharmacotherapeutic options. Pulm. Pharm. Ther. 18, 1-8.

Ross, S.M., 1971. A wavy wall analytic model of muco-ciliary pumping. Ph.D. Thesis. John Hopkins University.

Rubin, B.K., 2007. Mucus structure and properties in cystic fibrosis. Paed. Respir. Rev. $8,4-7$

Salathe, M., 2007. Regulation of mammalian ciliary beating. Ann. Rev. Physiol. 69, 401-422.

Sanderson, M.J., Sleigh, M.A., 1981. Ciliary activity of cultured rabbit tracheal epithelium: beat pattern and metachrony. J. Cell Sci. 47, 331-341.

Satir, P., Christensen, S.T., 2007. Overview of structure and function of mammalian cilia. Annu. Rev. Physiol. 69, 377-400.

Sleigh, M.A., Blake, J.R., Liron, N., 1988. The propulsion of mucus by cilia. Am. Rev. Respir. Dis. 137, 726-741.

Smith, D.J., 2006. Modelling muco-ciliary transport in the lung. Ph.D. Thesis. University of Birmingham, UK.

Smith, D.J., Gaffney, E.A., Blake, J.R., 2007a. Discrete cilia modelling with singularity distributions: application to the embryonic node and the airway surface liquid. Bull. Math. Biol. 69 (5), 1477-1510.

Smith, D.J., Gaffney, E.A., Blake, J.R., 2007b. A model of tracer transport in airway surface liquid. Bull. Math. Biol. 69 (3), 817-836.
Smith, D.J., Gaffney, E.A., Blake, J.R., 2007c. A viscoelastic traction layer model of muco-ciliary transport. Bull. Math. Biol. 69 (1), 289-327.

Tarran, R., Grubb, B.R., Gatzy, J.T., Davis, C.W., Boucher, R.C., 2001. The relative roles of passive surface forces and active ion transport in the modulation airway surface liquid volume and composition. J. Gen. Physiol. 118 (2), 223236.

Teff, Z., Priel, Z., Gheber, L.A., 2007. Forces applied by cilia measured on explants from mucociliary tissue. Biophys. J. 92, 1813-1823.

Teff, Z., Priel, Z., Gheber, L.A., 2008. The forces applied by cilia depend linearly on their frequency due to constant geometry of the effective stroke. Biophys. J. 94 298-305.

Verdugo, P., 1990. Goblet cells secretion and mucogenesis. Annu. Rev. Physiol. 52 157-176.

Weinbaum, S., Zhang, X., Han, Y., Vink, H., Cowin, S.C., 2003. Mechanotransduction and flow across the endothelial glycocalyx. Proc. Natl. Acad. Sci. U.S.A. 100 (13), 7988-7995.

Winet, H., 1987. The role of periciliary fluid in mucociliary flows: flow velocity profiles in frog palate mucus. Biorheology 24 (6), 635-642.

Winters, S.L., Davis, C.W., Boucher, R.C., 2007. Mechanosensitivity of mouse tracheal ciliary beat frequency: roles for $\mathrm{Ca} 20$, purinergic signaling, tonicity and viscosity. Am. J. Lung Cell. Mol. Physiol. 292, L614-L624.

Wolf, D.P., Blasco, L., Khan, M.A., Litt, M., 1977. Human cervical mucus. I. Rheologic characteristics. Fertil. Steril. 28 (1), 41-46. 